\title{
1 The unexpected extremophile: tolerance to fluctuating salinity in the green
}

\section{2 alga Picochlorum}

3

4 Fatima Foflonker ${ }^{1}$, Gennady Ananyev ${ }^{2,3}$, Huan Qiu $^{4}$, Andrenette Morrison ${ }^{5}$, Brian Palenik ${ }^{6}$, G.

5 Charles Dismukes ${ }^{2,3}$, and Debashish Bhattacharya ${ }^{4,5^{*}}$

6

$7{ }^{1}$ Department of Biochemistry and Microbiology, ${ }^{2}$ Waksman Institute of Microbiology,

$8{ }^{3}$ Department of Chemistry and Chemical Biology, ${ }^{4}$ Department of Ecology, Evolution and

9 Natural Resources, ${ }^{5}$ Department of Marine and Coastal Sciences, Rutgers University, NJ, USA,

$10{ }^{6}$ Scripps Institution of Oceanography, University of California, San Diego, La Jolla, CA, USA

$12{ }^{*}$ Corresponding author

13 Email: debash.bhattacharya@gmail.com

14 Tel: (848) 932-6218

15 Address:

16102 Foran Hall

1759 Dudley Rd

18 New Brunswick, NJ 08901-8520

19 USA 


\section{Abstract}

25 The broadly halotolerant green alga, Picochlorum strain SENEW3, has a highly reduced nuclear

26 genome of $13.5 \mathrm{Mbp}$ that encodes only 7,367 genes. It was originally isolated from a shallow,

27 mesophilic brackish-water lagoon that experiences extreme changes in temperature, light, and in

28 particular, salinity (freshwater to 3-fold seawater). We challenged Picochlorum cells with high or

29 low salinity shock and used transcriptomic and chlorophyll fluorescence analyses to elucidate

30 tolerance to salinity fluctuation. The transcriptome analysis showed that one-half of the coding

31 regions are differentially expressed in response to salinity changes. In addition, a significant

32 number of co-expressed genes (usually from different metabolic pathways) are co-localized in

33 the genome, forming 2-10 gene clusters. Whereas the overall salt stress response in Picochlorum

34 SENEW3 is similar to that in other salt-tolerant algae, the "operon-like" structure in this species

35 likely contributes to rapid recovery during salinity fluctuation. In summary, our work elucidates

36 how evolutionary forces play out in a streamlined genome. Picochlorum SENEW3 relies on a

37 broad array of adaptations from the reliance on horizontally transferred adaptive genes to the co-

38 localization of stress response genes and a robust photosystem II to deal with a fluctuating

39 environment. These attributes make Picochlorum SENEW3 of great biotechnological interest.

40

41 Keywords: Picochlorum; Chlorophyta; halotolerance; salt stress; co-localization; RNA-seq

43 Abbreviations: HGT, horizontal gene transfer; DE, differentially expressed; ASW, artificial

44 seawater; FRRF, fast repetition rate fluorometer; STF, single turnover flash; PSII, photosystem

45 II; WOC, water-oxidizing complex; $\mathrm{QF}$, quality factor; FDR, false discovery rate; $\mathrm{L}_{2} \mathrm{fc}, \log _{2}$ fold

46 change; TCA, tricarboxylic acid cycle; THF, tetrahydrofolate; LIN, lincomycin. 


\section{Introduction}

Most well known eukaryotic extremophiles are better described as polyextremophiles

49 because they survive multiple types of environmental stress. The sea ice diatom, Melosira

50 arctica, faces not only cold temperatures but also high $\mathrm{pH}$, irradiance stress, and fluctuating

51 salinity due to the brine habitat in the ice (Boetius et al., 2013). On the other end of the

52 temperature scale, Galdieria sulphuraria and Cyanidioschyzon merolae, red algal

53 thermoacidophiles native to hot spring environments, tolerate high levels of salinity and toxic

54 metal concentrations as well as low pH (Barbier et al., 2005). What is shared by these species are

55 highly reduced genomes and gene inventories (5-10 thousand genes) and functional

56 specialization (Wolf \& Koonin, 2013). In the case of G. sulphuraria, horizontal gene transfer

57 (HGT) and expansion of gene families with adaptive functions also have played key roles in their

58 evolutionary history (Qiu et al., 2013; Schönknecht et al., 2013). But are there environments that

59 are less exotic (i.e., mesophilic) that may pose just as much environmental risk? These habitats

60 could include the intertidal zone and shallow water lagoons, both of which endure high

61 ultraviolet and visible light levels, desiccation, and fluctuating salinity. To address this issue, we

62 studied Picochlorum sp. strain SENEW3 (SE3) (hereafter, Picochlorum SE3), a

63 "polyextremotolerant" green alga (Chlorophyta, Trebouxiophycae) that was isolated from a

64 shallow water estuary in San Diego County, California. This alga has one of the smallest

65 genomes known (13.5 Mbp) for a free-living eukaryote and encodes only 7,367 genes (Foflonker

66 et al., 2015). Nonetheless, Picochlorum SE3 is remarkably robust in the face of environmental

67 perturbations, thriving in freshwater as well as in 3-fold the salinity of seawater, light intensities

68 between $80-2000 \mu \mathrm{E} / \mathrm{m}^{2} / \mathrm{s}$, and temperatures that range from at least $16-33^{\circ} \mathrm{C}$ (Wang et al.,

69 2014). Picochlorum SE3 shows similarities in halotolerance range, environment, and other 
70 abiotic stress tolerances to the closely related species Picochlorum oklahomensis isolated from

71 the Great Salt Plains of Oklahoma (Henley et al., 2007).

72 Several other Picochlorum species including P. oklahomensis and P. atomus, have been

73 investigated and are considered suitable for biofuel, nutritional, nutraceutical, and waste water

74 remediation applications (De la Vega et al., 2011; Chen et al., 2012; Zhu, 2012; El-Kassas,

75 2013; Pereira et al., 2013; von Alvensleben et al., 2013; Zhu \& Dunford, 2013; 2014; Dahmen et

76 al., 2014). This is due to the robustness, ability to harvest through common flocculation methods

77 (Zhu, 2012), high biomass production (1.8-2.1 g/L maximum biomass) (De la Vega et al., 2011;

78 Zhu \& Dunford, 2013), high protein content (350-550 g/kg) (von Alvensleben et al., 2013; Zhu

79 \& Dunford, 2013; Dahmen et al., 2014), high carotenoid content (De la Vega et al., 2011), and

80 lipid accumulation (total lipid content reported to be 16-25\% dry weight, unstressed cells) (De la

81 Vega et al., 2011; Pereira et al., 2013; Zhu \& Dunford, 2013; Dahmen et al., 2014) exhibited by

82 members of this genus. The NAABB consortium reported a Picochlorum strain with the ability

83 to rapidly accumulate lipids under nitrogen depletion, accompanied with an increase in starch

84 accumulation, and demonstrated effective genetic manipulation in increasing lipid accumulation

85 by as much as $38 \%$ (Department of Energy, 2014). Salinity has also been reported as an effective

86 method of crop protection in reducing freshwater cyanobacterial contaminants in P. atomus

87 cultures (von Alvensleben et al., 2013). Therefore, Picochlorum SE3 may be of great

88 biotechnological interest due to its ability to withstand a hypervariable environment. This allows

89 the use of salinity as a crop protection mechanism and makes this alga potentially suitable for

90 large-scale open-pond cultivation.

91

92 2. Materials and Methods 


\subsection{Salinity shock experimental conditions}

94 Picochlorum sp. strain SE3 was cultivated in artificial seawater (Goldman \& McCarthy,

95 1978) based Guillard's f/2 medium (Guillard \& Ryther, 1962) containing $1 \mathrm{M} \mathrm{NaCl}$, without

96 silica (f/2 ASW -Si). Cultures were grown at $25^{\circ} \mathrm{C}$ under continuous light $\left(100 \mu \mathrm{E} \mathrm{m}^{-2} \mathrm{~s}^{-1}\right)$ on a

97 rotary shaker at $100 \mathrm{rpm}$ (Innova 43, New Brunswick Eppendorf). To determine growth rate,

98 cells adapted to $1 \mathrm{M} \mathrm{NaCl} \mathrm{f/2}$ media were inoculated in $\mathrm{f} / 2$ media containing various salt

99 concentrations and cell counts were performed using a hemacytometer (Neubauer improved,

100 Hausser Scientific), image capture (Infinity 2 camera, Lumenera corporation), and ImageJ

101 counting software. Growth rates were determined based on cell counts during exponential phase

102 using triplicate cultures (Equation 1).

103

$$
\text { growth rate }=\frac{2.303\left(\log \left(\text { count }_{2}\right)-\log \left(\text { count }_{1}\right)\right)}{\left(\text { time }_{2}-\text { time }_{1}\right)}
$$

105

106 For the transcriptome experiment, cells adapted to $1 \mathrm{M} \mathrm{NaCl}$ were pelleted, washed, and

107 inoculated in fresh $\mathrm{f} / 2$ media containing $1.5 \mathrm{M} \mathrm{NaCl}$ (high salt stress) and $10 \mathrm{mM} \mathrm{NaCl}$ (low salt

108 stress). $\mathrm{NaCl}$ was the only component of the f/2 ASW -Si media modified for all experiments.

109 Approximately $100 \mathrm{mg}$ of cells were harvested and flash frozen after $1 \mathrm{~h}$ and $5 \mathrm{~h}$ of incubation

110 with salt treatment under $100 \mu \mathrm{E} \mathrm{m}^{-2} \mathrm{~s}^{-1}$ light. Cells harvested prior to treatment were used as the

111 control. This experiment was performed in triplicate; i.e., three separate cultures were used for

112 each salinity condition and each of these was sampled at the $1 \mathrm{~h}$ and at $5 \mathrm{~h}$ time points.

113

114 2.2. Transcriptome sequencing 
115 Frozen algal cell samples were homogenized using the TissueLyser II (Qiagen) and total

116 RNA was extracted according to the RNeasy Plant Mini Kit (Qiagen) protocol. The cDNA

117 libraries were constructed using the TruSeq RNA Sample Preparation Kit (Illumina, San Diego,

118 CA) following the manufacturer's protocols. RNA concentrations were determined using a

119 NanoDrop 2000c Spectrophotometer (Thermoscientific) and Qubit 2.0 Fluorometer (Life

120 Technologies). Libraries were sequenced using the MiSeq Personal Genome Sequencer

121 (Illumina) with 2x80bp (paired end) and 1x160bp read lengths. See Table S1 for details. These

122 Illumina transcriptome data can be retrieved via NCBI BioProject RJNA245752 and are also

123 available at http://cyanophora.rutgers.edu/picochlorum/.

124

125 2.3. Transcriptome analysis

126 RNA-seq reads were trimmed and aligned to the Picochlorum SE3 genome using CLC

127 Genomics Workbench. Reads < 50bp in length were discarded during trimming and the RNA-

128 seq analysis in CLC was performed using default parameters. Differential expression was

129 determined using DESeq (R/bioconductor package) (Love et al., 2014) and read counts

130 determined by the CLC RNA-seq analysis as the input. A $P$-value of $1 \%$ and a $\log _{2}$ fold change

131 of 1 were set as the cutoffs for all differentially expressed (DE) genes discussed in this paper. DE

132 genes were used as input for the KEGG metabolic pathway mapping tool (Kanehisa et al., 2011)

133 to identify metabolic impacts of salinity stress. Gene ontology (GO) term enrichment analysis

134 was performed using the Fisher's exact test as part of the blast2go software (Conesa et al., 2005).

135 Differential gene expression overlap between treatments was determined with Venny 2.0

136 (Oliveros, 2007). The inference of protein targeting was done using TargetP (Emanuelsson et al.,

137 2000). 


\subsection{Co-localization analysis}

140 A co-regulated gene cluster was defined as two or more genes with shared properties

141 (e.g., up-regulation in response to salt treatment) that were physically linked. One or two

142 intervening genes (without the shared properties) between adjacent genes were allowed. Gene

143 clusters were merged when separated by two genes or less. To test if the observed clustering of

144 genes up-regulated by salt treatment was significantly more than expected by chance alone, we

145 removed genes derived from contigs encoding less than three genes. Clusters of up-regulated

146 genes were then identified as described above. We randomly sampled the same number of genes

147 as those actually up-regulated from the total gene population, and identified gene clusters using

148 this randomly sampled gene set. This random sampling-based analysis was repeated 1,000 times

149 and the information regarding resulting gene clusters was recorded. The gene cluster information

150 (the number of clusters and number of genes in clusters) derived from actual data and randomly

151 generated data were plotted as shown in supplementary information (SI) Fig. S4. The $p$-value of

152 actual gene number in a cluster (or cluster number) was defined as the number of random

153 samples generating a clustered gene number equal to or greater than the actual number divided

154 by the total random sample size (i.e., 1000).

155

\section{2.5. Photosynthetic measurements}

157 Chlorophyll fluorescence was measured in Fig. 1 using a fast repetition rate fluorometer

158 (FRRF) with saturated light pulses produced by a laser diode at an intensity of $32,000 \mu \mathrm{E} \mathrm{m} \mathrm{m}^{-2}$

159 with a $50 \mu$ s flash duration (defined as single turnover flash, STF), after 2 minutes of dark

160 incubation (Ananyev \& Dismukes, 2005). Mid-exponential phase cells adapted to $1 \mathrm{M} \mathrm{NaCl}$ were 
161 pelleted then shocked in media with various salinities. Aliquots and measurements were taken

162 hourly. The relative quantum efficiency of PSII charge separation (QY) was approximated by the

163 yield of variable chlorophyll fluorescence intensity $(F v / F m)$ (Kolber et al., 1998). Signal

164 averaging of four trains of 50 flashes was performed with 2 min dark pre-incubation between

165 each train. The steady-state value of $F v / F m$ was obtained by averaging the first 50 individual

166 STFs, denoted $F v / F m$, and is reported herein for three biological replicates. This average

167 eliminates the transient damping of period-four oscillations.

168 The relative fraction of photosystem II water-oxidizing complex (PSII-WOC) centers that

169 achieve productive water oxidation following primary charge separation was determined from

170 fits of the amplitude of the period-four oscillations of $F v / F m$ from individual STFs. The

171 oscillations from 50 STFs were fitted to a modified Kok model (VZAD model) to obtain the

172 inefficiency parameters describing the rate of damping and the S state populations in the dark

173 prior to flashing (Vinyard et al., 2013). By analogy with resonance circuit theory, the Quality

174 Factor $(\mathrm{QF})$ is used to quantify the efficiency of oscillations, defined as the inverse of the Kok

175 damping terms, $\mathrm{QF}=(\text { alpha }+ \text { beta })^{-1}$. Here, alpha represents misses $(\mathrm{STF}$ not resulting in

176 advancement of oxidation state of PSII-WOC) and beta represents double hits (STF resulting in

177 advancement by two oxidation states). Kok parameters were determined as an average of four

178 measurements. The $\mathrm{QF}$ is reported as an average of three biological replicates.

179 Photoinhibition was determined by tracking $F v / F m$ during exposure to $1500 \mu \mathrm{E} \mathrm{m}^{-2} \mathrm{~s}^{-1}$

180 high light conditions in the presence and absence of chloroplast protein synthesis inhibitor

181 lincomycin (LIN). Exponential phase cells adapted to $1 \mathrm{M} \mathrm{NaCl}$ were pelleted and then shocked

182 with various salinities with the addition of $10 \mathrm{mM}$ bicarbonate and $1 \mathrm{mM}$ LIN. $F v / F m$ was

183 measured using a PAM (pulse amplitude modulated) fluorometer (Photon Systems Instruments, 
184 Brno, Czech Republic) after 2-minute dark adaptation. Samples were exposed to high light for a

185 total of 70 minutes with measurements taken every ten minutes ( 7 min light exposure, 2 min dark

186 adaptation, 1 minute measurement time). Experiments were performed with three biological

187 triplicates. The PSII inactivation rate was calculated by fitting $F v / F m$ over time to a

188 biexponential decay function using the OriginPro 2014 software (OriginLab). The lifetimes of

189 the biexponential curves were calculated using the fitting parameters (Fig. 4) and equation 2:

$$
\text { lifetime }=(A 1 \times t 1)+(A 2+t 2)
$$

\section{3. Results and Discussion}

\section{3.1. High and low salinity stress elicits separate metabolic responses}

194 We determined the appropriate conditions to study short-term response to salt stress in

195 Picochlorum SE3. The growth rate and average PSII quantum efficiency (proportional to $F v / F m$ )

196 were used to assess the effects of various salt concentrations on $1 \mathrm{M} \mathrm{NaCl}$ pre-adapted cultures of

197 the alga (Fig. 1). The time dependence of $F v / F m$ over $24 \mathrm{~h}$ shows that the PSII quantum yield,

198 which is a measure of the light energy conversion to heat + photochemical water oxidation, is

199 diminished at both lower and higher salt concentrations. The salt dependence of $F v / F m$ after $24 \mathrm{~h}$

200 follows a similar sequence as the growth rate, indicating that energy resources that normally go

201 into growth are diverted to the maintenance of osmotic balance, with both hypo- and hyper-

202 osmotic balance diverting energy resources. Higher growth rates at lower salinities ( 0.1 and

$2030.01 \mathrm{M})$ than the pre-adapted condition (1M) may simply indicate that lower salinity results in

204 optimum growth over the period of several days. However, Fig. 1 indicates that lower salinities

205 have similar effects as high salinities in the short-term. When examined in more detail it is

206 apparent that the kinetic response of $F v / F m$ differs for hypo- vs. hyper-osmotic changes. The 
207 kinetics of recovery are faster when the change in salt concentration is smallest for both hypo-

208 and hyper-osmotic changes. Additionally, all of the hyper-salinity samples (1M, 1.5M, and 1.6

$209 \mathrm{M} \mathrm{NaCl})$ attain a steady-state between $7 \mathrm{~h}$ and $24 \mathrm{~h}$, whereas the hypo-salinity samples $(0.1 \mathrm{M}$,

$2100.01 \mathrm{M}$, and $0 \mathrm{M} \mathrm{NaCl}$ ) are still recovering at $24 \mathrm{~h}$, indicated by the positive slopes. This

211 difference may be simply because the changes in concentration are so much larger (10X and

212 100X), thereby slowing recovery. No large-scale cell rupture due to osmotic shock was observed

213 microscopically, but smaller-scale rupture could have gone unnoticed due to the small cell size

$214 \quad(2-3 \mu \mathrm{m})$ of this coccoid alga.

215 For the transcriptome analysis we chose $1.5 \mathrm{M}$ and $10 \mathrm{mM} \mathrm{NaCl}$ concentrations to

216 represent hyper- and hypo-salinity stresses because both elicited similar photosynthetic

217 efficiency responses in the short-term and exhibited some recovery of growth rate under long-

218 term acclimation. The $1 \mathrm{M}$ to $10 \mathrm{mM}$ stress represents what Picochlorum cells might experience

219 during a rain event in its natural environment. Transcriptomic analysis reveals that salt stress

220 induces significant gene expression changes in Picochlorum SE3 with a total of 3,681 genes

221 (50\% of the nuclear gene inventory) being differentially expressed (DE; $>1 \log _{2}$ fold change

222 [hereafter, $\left.\left.\mathrm{L}_{2} \mathrm{fc}\right]\right), p<0.01$, adjusted for the false discovery rate, FDR, of $5 \%$ ) by salt stress over

223 the 5-hour time course, relative to the pre-stress control. This includes 12 of 24 genes that

224 originated in Picochlorum SE3 via HGT from bacterial sources (SI Table S2) (Foflonker et al.,

225 2015), suggesting functional relevance of these foreign genes in salt tolerance, analogous to what

226 was found in G. sulphuraria (Qiu et al., 2013; Schönknecht et al., 2013). More genes showed

227 differential expression at $1 \mathrm{~h}(3,256)$ than at $5 \mathrm{~h}(1,629)$ and around one-half of the DE genes at $1 \mathrm{~h}$

228 returned to below threshold levels $\left(<1\right.$ and $\left.<-1 \mathrm{~L}_{2} \mathrm{fc}\right)$ by the $5 \mathrm{~h}$ time point $(77 \% \mathrm{high}, 68 \%$ low

229 salinity) (SI Fig. S1, S2). In addition, each salinity treatment resulted in different DE gene sets, 
230 with some statistically significant overlap of genes involved in multiple treatments (SI Fig. S1,

231 Table S3; the full list of DE genes is shown SI Table S4). For example, the 1h up-regulated gene

232 set shared 173 genes, with 742 and 722 genes belonging uniquely to the high and low salinity

233 responses, respectively. In terms of biochemical pathways, there is little overlap in the initial

234 high and low salinity responses, indicating two very different types of stress on the cell (Fig. 3,

235 SI Fig. S3). We posit that high and low salinity shock, and salt acclimation all represent different

236 challenges to Picochlorum SE3, which deals with them using specialized gene sets.

238 3.2. Co-localization of co-expressed genes in response to salt shock

239 For each culture treatment, we searched for clusters of co-localized DE genes with a

240 shared expression pattern (i.e., co-expressed), allowing for a maximum of two intervening genes

241 not sharing that expression pattern (Fig. 2a). Randomization analysis was performed to

242 determine the statistical significance of these putative clusters (SI Fig. S4). These results indicate

243 that the number of genes in clusters is statistically significant in most data sets, meaning that

244 larger clusters of co-localized genes are found within co-expressed data sets than could be

245 attributed to chance alone. Under some conditions, the number of clusters formed is also

246 significant. These results hold true for the majority of the conditions, even when more stringent

247 gene expression cutoffs are used (1.5 vs. $1.0 \mathrm{~L}_{2} \mathrm{fc}$ ) (SI Table S5, Fig. 2a). Allowing for three

248 intervening genes resulted in loss of statistical significance in clustering. The underlying

249 transcription control mechanisms of these clusters are, however, unknown.

250 The co-localized genes comprise 2-10 gene clusters and constitute $42-72 \%$ of the total

251 number of genes in individual co-expressed data sets ( $1.0 \mathrm{~L}_{2} \mathrm{fc}$ cutoff). The results shown in Fig.

2522 demonstrate that more co-expressed genes, both up- and down-regulated and at both salinities, 
253 are co-localized at $1 \mathrm{~h}$ when compared to the $5 \mathrm{~h}$ time point. For example, 61.6\% (546/886) of

254 genes form clusters under high salinity at $1 \mathrm{~h}$ compared to $42.4 \%(225 / 531)$ at the $5 \mathrm{~h}$ time point

255 (1.0 $\mathrm{L}_{2} \mathrm{fc}$ cutoff). This suggests that genes are organized in close proximity in the genome for

256 more efficient regulation of gene expression during the initial phase of salinity shock. Although

257 these clusters of co-localized and co-expressed genes are functionally related in that they all take

258 part in the salt stress response, the vast majority do not appear to be members of the same

259 biochemical pathway. Exceptions include genes involved in urea degradation, nitrate

260 assimilation, acetate assimilation, pyruvate to acetoin conversion, some light regulated genes,

261 and photorespiration (SI Table S6). Interestingly, many of the co-localized genes in Picochlorum

262 SE3 are not physically linked in the genomes of other green algae such as Chlorella vulgaris and

263 Coccomyxa subellipsoidea (SI Table S6; full output presented in SI Table S7). Some clusters,

264 including genes in the nitrate assimilation pathway, however, are partially conserved in other

265 green algae including, C. vulgaris, C. subellipsoidea, and C. reinhardtii (Quesada et al., 1998).

266 The cluster of photorespiration related genes appear to be conserved in C. subellipsoidea as well.

267 These results suggest that stress-related genes in streamlined eukaryotic genomes may be

268 organized in operon-like structures similar to bacteria. This presumably allows rapid activation

269 under stressful conditions. However, no evidence of polycistronic transcription was found in

270 Picochlorum SE3.

271

272 3.3. High photorespiration influences carbon and nitrogen flux at high salinity

273 The most striking change in response to high salinity shock at $1 \mathrm{~h}$ is the up-regulation of

274 genes involved in photorespiration (Fig. 3; SI Table S8), which may function in stress protection

275 (Wingler et al., 2000). Photorespiration in green algae such as $C$. reinhardtii differs from that in 
276 plants, in that glycolate is converted to glyoxylate via glycolate dehydrogenase in the

277 mitochondrion rather than via glycolate oxidase in the peroxisome (Stabenau \& Winkler, 2005).

278 In traditional photorespiration, $\mathrm{H}_{2} \mathrm{O}_{2}$ is a byproduct of the glycolate oxidase reaction, resulting in

279 an increased need for catalase activity. Up-regulation of glycolate dehydrogenase and down-

280 regulation of glycolate oxidase and catalase suggests that the mechanism of photorespiration in

281 Picochlorum SE3 is similar to that of other green algae. Glycolate dehydrogenase is also located

282 in a cluster with two other genes in the photorespiration pathway (SI Table S6).

283 Reduced $\mathrm{CO}_{2}$ concentrations at high salinities can inhibit the preferred photosynthetic

284 carbon fixation pathway of Rubisco, stimulating the competing pathway of photorespiration.

285 Conditions of $\mathrm{CO}_{2}$ limitation are also correlated with increased carbon concentrating activities

286 via up-regulation of carbonic anhydrase in Dunaliella salina; i.e., to increase photosynthetic

287 carbon fixation through the Calvin-Benson-Bassham cycle (Booth \& Beardall, 1991; Fisher et

288 al., 1996). Picochlorum SE3 also displays this response with strong up-regulation of carbonic

289 anhydrase under high salinity stress. Carbon flux through the TCA cycle is down-regulated at $1 \mathrm{~h}$,

290 potentially due to the inhibition of reductant production via the TCA cycle due to excessive

291 NADH produced during the glycine to serine conversion in photorespiration (Bykova et al.,

292 2005) (Fig. 3, SI Fig. S5). One-carbon metabolism including tetrahydrofolate (THF) synthesis

293 from glycine and the interconversion between its derivatives is also highly up-regulated under

294 high salinity. In Arabidopsis, THF metabolism is essential to photorespiration, thereby

295 maintaining carbon fixation under $\mathrm{CO}_{2}$-limited conditions (Collakova et al., 2008).

296 High photorespiration rates in plants result in the generation of ammonia as a byproduct

297 of the conversion of glycine to serine, which is subsequently converted to glutamate

298 (Rachmilevitch et al., 2004). Accordingly, one copy each of glutamine synthetase and 
299 ferrodoxin-dependent glutamate synthase, the major pathway for ammonia fixation, is up-

300 regulated in Picochlorum SE3 under high salinity stress. Nitrate and urea assimilation are also

301 significantly up-regulated, potentially serving as nitrogen sources for the increased protein

302 synthesis demands under salinity stress. The opposite is true for nitrogen assimilation at low

303 salinity stress, however, translation and ribosome biogenesis are up-regulated. These results

304 again indicate that Picochlorum SE3 cells are less stressed at low (versus high) salinity.

306 Starch and osmolytes

Proline is the major osmolyte in Picochlorum oklahomensis; glycerol and

308 glucosylglycerol were detected to a lesser extent (Henley et al., 2004). One gene involved in

309 proline synthesis was up-regulated in Picochlorum SE3 under high salinity, and an increased

310 expression of pathways leading towards glutamate synthesis, the precursor of proline, was

311 observed. In Dunaliella, starch formation decreases and starch degradation increases as salinity

312 increases in favor of glycerol synthesis (Goyal, 2007; Xia et al., 2014). In contrast, starch

313 synthesis is up-regulated and starch degradation is down-regulated, whereas no evidence of

314 increased glycerol synthesis was observed in Picochlorum SE3 under the high salt conditions

315 used in this experiment. Synthesis of trehalose, another osmolyte, is up-regulated at $1 \mathrm{~h}$ and

316 sorbitol is up-regulated at $5 \mathrm{~h}$. As expected, in order to maintain osmotic equilibrium at low

317 salinity, osmolyte synthesis is down-regulated, and accordingly starch synthesis is up-regulated.

318 The up-regulation of starch synthesis under both high and low salinity stress conditions may

319 indicate that proline rather than glycerol is the major osmolyte in Picochlorum SE3. Other

320 aspects of the stress response in Picochlorum SE3 (e.g., cell wall and membrane remodeling) are

321 discussed in the supplementary information Notes S1. 


\section{3.4. Response of the photosynthetic machinery}

324 Photoinhibition involves light-induced oxidative damage to the PSII protein D1 and

325 inactivation of the WOC (Murata et al., 2007; Kolling et al., 2009). PSII repair entails D1

326 protein digestion by internal proteases and replacement of the damaged protein via de novo

327 synthesis (Dasgupta et al., 2008). Photoinhibition, when accelerated by environmental stresses,

328 including salinity, reduces photosynthetic carbon fixation, which accelerates light-driven $\mathrm{H}_{2} \mathrm{O}_{2}$

329 generation, which in turn accelerates D1 protein damage (Neale \& Melis, 1989; Allakhverdiev \&

330 Murata, 2004; Takahashi \& Murata, 2008; Kolling et al., 2009). We measured the rate of

331 photoinhibition under high light, $1500 \mu \mathrm{E} \mathrm{m}^{-2} \mathrm{~s}^{-1}$ (i.e., different from the transcriptome

332 experimental conditions), at various salinities in the presence and absence of the chloroplast

333 protein synthesis inhibitor lincomycin (LIN). These photoinhibition curves (Fig. 4) are the result

334 of several interacting phenomena: photoprotection, D1 biosynthesis de novo, and osmoregulation

335 after salinity shock. They may be fitted via biexponential decrease of $F v / F m$, with the two phases

336 distinguished by an initial period of osmoregulation and a later phase dominated by D1

337 biosynthesis. Both high and low salinity shock conditions exhibit a similar pattern of reduced

338 decline of D1 protein synthesis compared to the control $(1 \mathrm{M} \mathrm{NaCl}) ; 10 \mathrm{mM} \mathrm{NaCl}$ shows the

339 shortest difference in lifetimes between samples with and without LIN (1.6 min) (Fig. 4a)

340 compared to the control (16.9 $\mathrm{min}), 1.5 \mathrm{M} \mathrm{NaCl}(6.1 \mathrm{~min})$, and $0.4 \mathrm{M} \mathrm{NaCl}(6.8 \mathrm{~min})$ conditions.

341 We postulate that the additional stress of salinity shock diverts resources from D1 protein repair

342 to the energy intensive process of maintaining cell homeostasis during salt shock. These results

343 partially support the hypothesis that high salinity may provide protection against other abiotic

344 stresses such as irradiance and temperature. This phenomenon has been reported for other 
345 halotolerant green algae, such as Nannochloris sp. and Dunaliella parva (Henley et al., 2002).

346 However, because our experiments examined salinity shock, the observed lessened stress effects

347 under $0.4 \mathrm{M}$ and $1 \mathrm{M} \mathrm{NaCl}$ and high light may be better explained by acclimation.

348 SI Fig. S7 highlights the faster recovery of the WOC cycling efficiency, indicated by the

349 quality factor $(\mathrm{QF})$ at low salinity shock versus high salinity shock (halftime $=0.5 \mathrm{~h}$ at $10 \mathrm{mM}$

$350 \mathrm{NaCl}$ and $4.5 \mathrm{~h}$ at $1.5 \mathrm{M} \mathrm{NaCl})$. Recovery of WOC cycling efficiency appears to correlate with

351 recovery of $F v / F m$ or overall photosynthetic efficiency at high salinity, but recovers faster than

$352 F v / F m$ at low salinity. Whereas, D1 repair is equally inhibited by the low and high salinity

353 conditions, WOC efficiency may provide a greater contribution to photodamage at high

354 salinities.

355

\section{4. Conclusions}

357 Our genome-wide and PSII analyses demonstrate that despite living in an apparently

358 mesophilic lagoon environment, environmental fluctuations have left significant "footprints" on

359 the Picochlorum SE3 genome. Diametrically opposed salinity conditions impose distinct

360 challenges that this alga responds to with specialized gene sets. Shock and acclimated responses

361 also differ, highlighting the challenges posed by a rapid versus gradual environmental change

362 with respect to salinity. Overall, Picochlorum SE3 responds to high/low salinity stress in a

363 similar fashion as other algae and plants; i.e., photoprotective mechanisms, oxidative stress

364 response, cell wall and membrane rearrangement, nitrogen assimilation, and diverting resources

365 from growth and PSII repair in favor of maintaining homeostasis. Despite these shared

366 responses, the key to the ability of Picochlorum SE3 to withstand massive environmental

367 fluctuations is likely explained by genome organization. Co-localization of genes within 
368 specialized gene sets appears to function similarly to bacterial operons in enhancing a rapid

369 response to shock. In summary, our study highlights a compact genome that has evolved a broad

370 array of adaptations from HGT to co-localization of stress response genes to a robust PSII to deal

371 with a challenging environment. Although our results suggest that energetic resources are

372 diverted from growth and productivity during periods of salinity shock in favor of maintaining

373 cell homeostasis, Picochlorum SE3 is highly adapted to rapid acclimatation (beginning within 5

374 hours of salinity shock) to salinity shock and maintains growth rates over a broad range of

375 salinities. Therefore, Picochlorum SE3 may be suitable for open-pond cultivation under

376 conditions of high irradiance and high salinity, which would not be tolerable to competing

377 freshwater and many marine microalgal species. Its broad range of salinity and shock tolerance

378 makes Picochlorum SE3 particularly suited to cultivation in seawater or saline groundwater

379 because it can tolerate evaporative loss in an open-pond environment.

\section{5. Acknowledgements}

This work was supported by grants from the Department of Energy (DE-EE0003373/001)

383 to D.B. and B.P., a grant from the DOE Office of Basic Energy Sciences (DE-FG02-

384 10ER16195) to G.C.D., and graduate training support from the National Science Foundation

385 IGERT for Renewable and Sustainable fuels program at Rutgers University (0903675) to F.F.

386 We are grateful to the Rutgers University School of Environmental and Biological Sciences and

387 members of the Genome Cooperative at SEBS for supporting this research. F.F. was aided by an

$388 R$ workshop sponsored by the Porphyra Algal Genomics RCN (NSF0741907).

\section{References}


391 Allakhverdiev SI, Murata N. 2004. Environmental stress inhibits the synthesis de novo of proteins involved in the photodamage-repair cycle of photosystem II in Synechocystis sp. PCC 6803. Biochimica et Biophysica Acta (BBA)-Bioenergetics 1657(1): 23-32.

Ananyev G, Dismukes GC. 2005. How fast can photosystem II split water? Kinetic performance at high and low frequencies. Photosynthesis Research 84(1-3): 355365.

Barbier G, Oesterhelt C, Larson MD, Halgren RG, Wilkerson C, Garavito RM, Benning C, Weber AP. 2005. Comparative genomics of two closely related unicellular thermo-acidophilic red algae, Galdieria sulphuraria and Cyanidioschyzon merolae, reveals the molecular basis of the metabolic flexibility of Galdieria sulphuraria and significant differences in carbohydrate metabolism of both algae. Plant Physiology 137(2): 460-474.

Boetius A, Albrecht S, Bakker K, Bienhold C, Felden J, Fernández-Méndez M, from the melting Arctic sea ice. Science 339(6126): 1430-1432. anhydrase activity in the halotolerant alga Dunaliella salina (Chlorophyta). Phycologia 30(2): 220-225. photorespiration and respiration in transgenic potato plants with antisense reduction in glycine decarboxylase. Planta 222(1): 130-140. 
413 Chen T-Y, Lin H-Y, Lin C-C, Lu C-K, Chen Y-M. 2012. Picochlorum as an alternative to

$414 \quad$ Nannochloropsis for grouper larval rearing. Aquaculture 338(Journal Article): 82-88.

415 Collakova E, Goyer A, Naponelli V, Krassovskaya I, Gregory JF, Hanson AD, Shachar-

416 Hill Y. 2008. Arabidopsis 10-formyl tetrahydrofolate deformylases are essential for

417 photorespiration. The Plant Cell Online 20(7): 1818-1832.

418 Conesa A, Götz S, García-Gómez JM, Terol J, Talón M, Robles M. 2005. Blast2GO: a

419 universal tool for annotation, visualization and analysis in functional genomics

$420 \quad$ research. Bioinformatics 21(18): 3674-3676.

421 Dahmen I, Chtourou H, Jebali A, Daassi D, Karray F, Hassairi I, Sayadi S, Abdelkafi S,

422 Dhouib A. 2014. Optimisation of the critical medium components for better growth

423 of Picochlorum sp. and the role of stressful environments for higher lipid production.

$424 \quad$ Journal of the Science of Food and Agriculture 94(8): 1628-1638.

425 Dasgupta J, Ananyev GM, Dismukes GC. 2008. Photoassembly of the water-oxidizing

426 complex in photosystem II. Coordination Chemistry Reviews 252(3): 347-360.

427 De la Vega M, Díaz E, Vila M, León R. 2011. Isolation of a new strain of Picochlorum sp.

428 and characterization of its potential biotechnological applications. Biotechnology

429 Progress 27(6): 1535-1543.

430 Department of Energy. 2014. National alliance for advanced biofuels and bio-products

$431 \quad$ (NAABB) final report

432 El-Kassas HY. 2013. Growth and fatty acid profile of the marine microalga Picochlorum Sp.

433 grown under nutrient stress conditions. The Egyptian Journal of Aquatic Research

$434 \quad 39(4): 233-239$. 
435 Emanuelsson 0, Nielsen H, Brunak S, von Heijne G. 2000. Predicting subcellular

436

437

438

439

440

441

442

443

444

445

446

447

448

449

450

451

452

453

454

455

456

457

localization of proteins based on their N-terminal amino acid sequence.J Mol Biol 300(4): 1005-1016.

Fisher M, Gokhman I, Pick U, Zamir A. 1996. A salt-resistant plasma membrane carbonic anhydrase is induced by salt in Dunaliella salina. Journal of Biological Chemistry 271(30): 17718-17723.

Foflonker F, Price DC, Qiu H, Palenik B, Wang S, Bhattacharya D. 2015. Genome of the halotolerant green alga Picochlorum sp. reveals strategies for thriving under fluctuating environmental conditions. Environmental Microbiology 17(2): 412-426.

Goldman JC, McCarthy JJ. 1978. Steady state growth and ammonium uptake of a fast growing marine diatom 1. Limnology and Oceanography 23(4): 695-703.

Goyal A. 2007. Osmoregulation in Dunaliella, Part II: Photosynthesis and starch contribute carbon for glycerol synthesis during a salt stress in Dunaliella tertiolecta. Plant Physiology and Biochemistry 45(9): 705-710.

Guillard RRL, Ryther JH. 1962. Studies of Marine Planktonic Diatoms: I. Cyclotella nana Hustedt and Detonula confervacea (Cleve) Gran. Canadian Journal of Microbiology 8(2): 229-239.

Henley WJ, Hironaka JL, Guillou L, Buchheim MA, Buchheim JA, Fawley MW, Fawley KP. 2004. Phylogenetic analysis of the'Nannochloris-like'algae and diagnoses of Picochlorum oklahomensis gen. et sp. nov.(Trebouxiophyceae, Chlorophyta). Phycologia 43(6): 641-652.

Henley WJ, Kvíderová J, Kirkwood AE, Milner J, Potter AT 2007. Life in a Hypervariable Environment. Algae and Cyanobacteria in Extreme Environments: Springer, 681-694. 
458 Henley WJ, Major KM, Hironaka JL. 2002. Response to Salinity and heat stres in two halotolerant chlorophyte algae. Journal of Phycology 38(4): 757-766.

460 Kanehisa M, Goto S, Sato Y, Furumichi M, Tanabe M. 2011. KEGG for integration and 461 interpretation of large-scale molecular data sets. Nucleic Acids Res: gkr988.

462 Kolber ZS, Prášil O, Falkowski PG. 1998. Measurements of variable chlorophyll 463 fluorescence using fast repetition rate techniques: defining methodology and

Murata N, Takahashi S, Nishiyama Y, Allakhverdiev SI. 2007. Photoinhibition of

Kolling DR, Brown TS, Ananyev G, Dismukes GC. 2009. Photosynthetic oxygen evolution experimental protocols. Biochimica et Biophysica Acta (BBA)-Bioenergetics 1367(1): 88-106.

is not reversed at high oxygen pressures: mechanistic consequences for the wateroxidizing complex. Biochemistry 48(6): 1381-1389. photosystem II under environmental stress. Biochimica et Biophysica Acta (BBA)Bioenergetics 1767(6): 414-421.

Neale PJ, Melis A. 1989. Salinity-stress enhances photoinhibition of photosynthesis in Chlamydomonas reinhardtii. Journal of Plant Physiology 134(5): 619-622.

474 Oliveros JC. 2007. VENNY. An interactive tool for comparing lists with Venn Diagrams.

Pereira H, Barreira L, Custódio L, Alrokayan S, Mouffouk F, Varela J, Abu-Salah KM, Ben-Hamadou R. 2013. Isolation and fatty acid profile of selected microalgae strains from the Red Sea for biofuel production. Energies 6(6): 2773-2783. 2013. Adaptation through horizontal gene transfer in the cryptoendolithic red alga Galdieria phlegrea. Current Biology 23(19): R865-R866. 
481 Quesada A, Hidalgo J, Fernandez E. 1998. Three Nrt2 genes are differentially regulated in Chlamydomonas reinhardtii. Molecular and General Genetics MGG 258(4): 373-377.

483 Rachmilevitch S, Cousins AB, Bloom AJ. 2004. Nitrate assimilation in plant shoots depends on photorespiration. Proceedings of the National Academy of Sciences, USA 101(31): 11506-11510.

Schönknecht G, Chen W-H, Ternes CM, Barbier GG, Shrestha RP, Stanke M, Bräutigam

A, Baker BJ, Banfield JF, Garavito RM. 2013. Gene transfer from bacteria and archaea facilitated evolution of an extremophilic eukaryote. Science 339(6124): 1207-1210.

Stabenau H, Winkler U. 2005. Glycolate metabolism in green algae. Physiologia Plantarum 123(3): 235-245.

Takahashi S, Murata N. 2008. How do environmental stresses accelerate photoinhibition? Trends in Plant Science 13(4): 178-182.

494 Vinyard DJ, Zachary CE, Ananyev G, Dismukes GC. 2013. Thermodynamically accurate modeling of the catalytic cycle of photosynthetic oxygen evolution: A mathematical solution to asymmetric Markov chains. Biochimica et Biophysica Acta (BBA)Bioenergetics 1827(7): 861-868.

von Alvensleben N, Stookey K, Magnusson M, Heimann K. 2013. Salinity tolerance of picochlorum atomus and the use of salinity for contamination control by the freshwater cyanobacterium Pseudanabaena limnetica. Plos One 8(5): e63569.

Wang S, Lambert W, Giang S, Goericke R, Palenik B. 2014. Microalgal assemblages in a poikilohaline pond. Journal of Phycology 50(2): 303-309. 
503 Wingler A, Lea PJ, Quick WP, Leegood RC. 2000. Photorespiration: metabolic pathways

504 and their role in stress protection. Philosophical Transactions of the Royal Society B:

$505 \quad$ Biological Sciences 355(1402): 1517-1529.

506 Wolf YI, Koonin EV. 2013. Genome reduction as the dominant mode of evolution.

$507 \quad$ Bioessays 35(9): 829-837.

508 Xia B-B, Wang S-H, Duan J-B, Bai L-H. 2014. The relationship of glycerol and glycolysis

509 metabolism patway under hyperosmotic stress in Dunaliella salina. Central

$510 \quad$ European Journal of Biology 9(9): 901-908.

511 Zhu Y. 2012. Biomass and flocculation characteristics of Picochlorum oklahomensis and

$512 \quad$ Nannochloropsis oculata: Oklahoma State University.

513 Zhu Y, Dunford NT. 2013. Growth and biomass characteristics of Picochlorum

$514 \quad$ oklahomensis and Nannochloropsis oculata. Journal of the American Oil Chemists'

$515 \quad$ Society(Journal Article): 1-9.

516

$517 \quad$ Figure Legends

518 Fig. 1. (a) Average chlorophyll variable fluorescence yield $(F v / F m)$ and (b) Growth rate of the

519 algal cultures acclimated to $1 \mathrm{M} \mathrm{NaCl}$ media, following transfer to media containing various salt

520 concentrations under $100 \mu \mathrm{E} \mathrm{m}^{-2} \mathrm{~s}^{-1}$ light. $F v / F m$ is the average of 4 trains of 50 STFs and three

521 biological replicates. Error bars represent standard deviation.

522

523 Fig. 2. (a) Examples of co-expressed and co-localized gene clusters. Gene clusters, as defined in

524 the text, are denoted with boxes. Blue represents up-regulated genes; red, down-regulated; gray,

525 not DE. The numbers above the boxes show relative gene locations within contigs; gene numbers 
526 are labeled above the boxes. (b) Number of genes co-localized versus total genes in gene set

527 (percentages labeled) at the 1.0 and $1.5 \mathrm{~L}_{2} \mathrm{fc}$ cutoffs. Only statistically significant results are

528 shown (see SI Table S5 for details).

529

530 Fig. 3. Summary of the salt shock response in Picochlorum SE3 at $1 \mathrm{~h}$ under (a) high salinity and

531 (b) low salinity conditions. Genes/pathways in blue are up-regulated (>1.0 $\left.\mathrm{L}_{2} \mathrm{fc}\right)$; red, down-

532 regulated $\left(<-1.0 \mathrm{~L}_{2} \mathrm{fc}\right)$; gray, not $\mathrm{DE}\left(<1.0 \mathrm{~L}_{2} \mathrm{fc}\right.$ and $\left.>-1.0 \mathrm{~L}_{2} \mathrm{fc}\right)$. Solid colored arrows indicate

533 that at least one copy of the gene is DE (i.e. other copies may not be DE), whereas arrows with a

534 blue/red gradient have gene copies that are both up- and down-regulated. The numbers

535 correspond to enzymes shown in Table S8. G3P, glyceraldehyde 3-phosphate; DHAP,

536 dihydroxyacetone phosphate; DHA, dihydroxyacetone; THF, tetrahydrofolate; SQDG,

537 sulfoquinovosyl diacylglycerol; UDP-GlcNAc, uridine diphosphate N-acetylglucosamine;

538 geranylgeranyl-PP, geranylgeranyl pyrophosphate.

539

540 Fig. 4. Photoinhibition under $1500 \mu \mathrm{E} \mathrm{m}^{-2} \mathrm{~s}^{-1}$ high light conditions in the presence and absence

541 of chloroplast protein synthesis inhibitor lincomycin (LIN). Cells adapted to $1 \mathrm{M} \mathrm{NaCl}$ media

542 were incubated in media at various salinities. The data are fit to a second order exponential decay

543 curve. The relative amplitudes (A1, A2) and lifetimes in minutes ( $\mathrm{t} 1, \mathrm{t} 2)$ values are shown. Error

544 bars represent standard deviation.

545

546 The following Supporting Information is available for this article:

547 Notes S1. Salinity stress response in Picochlorum SE3 
548 Fig. S1. Venn diagram comparing differentially expressed genes at both salinities and time

549 points

550 Fig. S2. Gene expression patterns at high and low salinity over the time course

551 Fig. S3. KEGG metabolic map overview comparing the low and high salinity stress response

552 Fig. S4. Example of a randomization analysis that simulates gene clustering.

553 Fig. S5. KEGG pathway analysis of genes involved in the TCA cycle comparing high and low

554 salinity shock

555 Fig. S6. KEGG pathway analysis of genes involved in protein processing in the endoplasmic

556 reticulum comparing high and low salinity shock

557 Fig. S7. Effect of salinity on quality factor (QF)

558 Table S1. Number of RNA-seq reads sequenced.

559 Table S2. Differentially expressed genes of bacterial origin.

560 Table S3. Excel file of shared differentially expressed genes between the four conditions tested.

561 Table S4. Excel file of all DE genes. Highlighted cells (orange) denote HGT.

562 Table S5. Summary of number of co-expressed co-localized genes forming clusters.

563 Table S6. Examples of co-expressed co-localized gene clusters

564 Table S7. Excel file of all co-expressed co-localized genes.

565 Table S8. Accompanying table for Fig. 3 with gene expression values of differentially expressed 566 genes. 
Figure 1

(a)

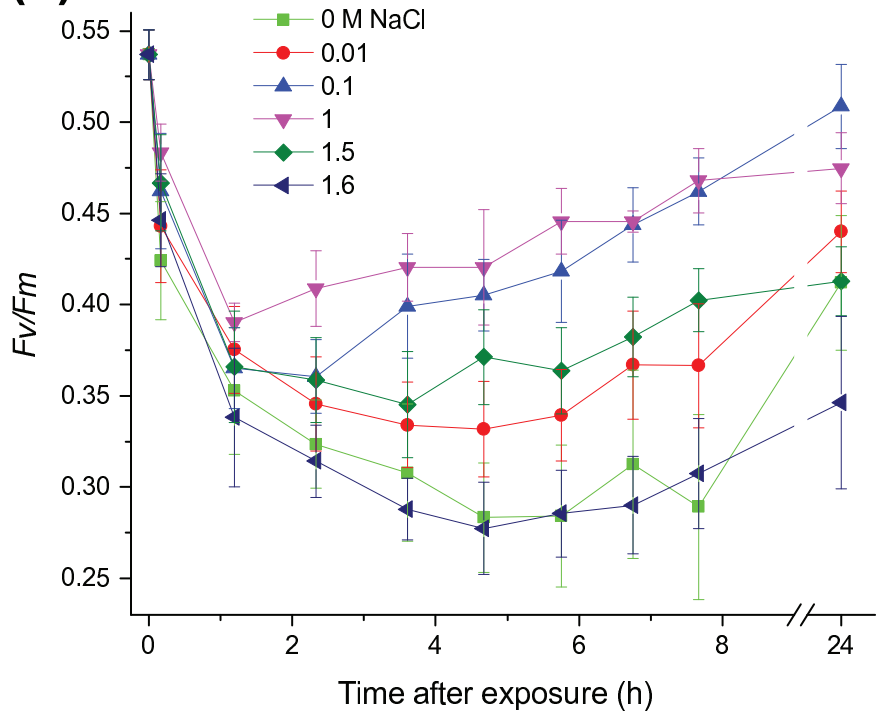

(b)

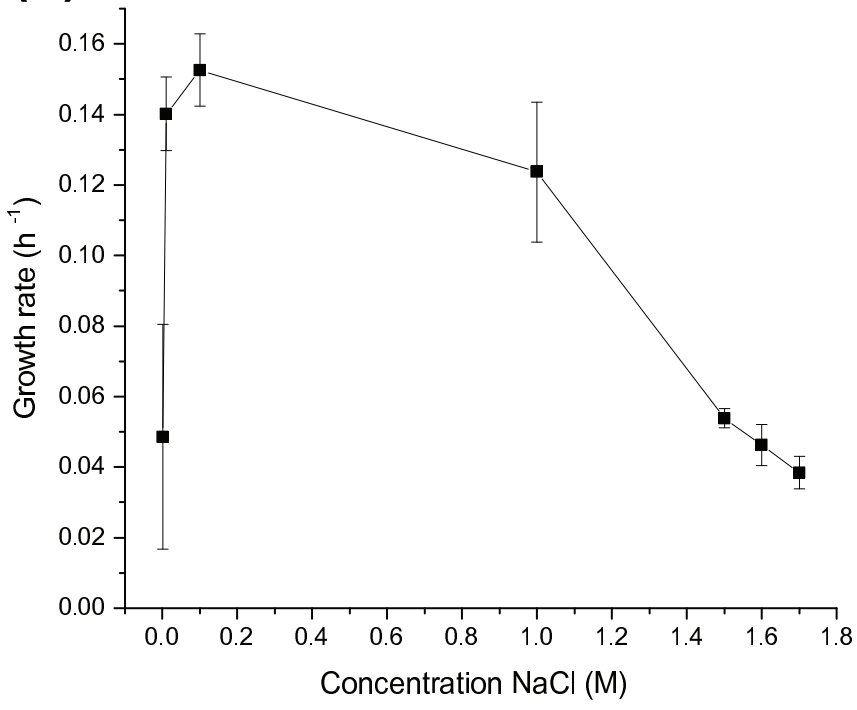


(a) bp position on contig $135,000 \quad 140,000 \quad 145,000 \quad 150,000 \quad 15,000 \quad 160,000 \quad 165,000 \quad 170,000 \quad 175,000 \quad 180,000$ High salinity
$1 \mathrm{~h}$

96.g445 $1 \mathrm{~h}$
(1) $\square \square \square$
96.9450

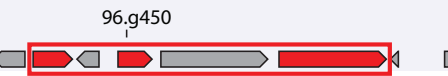

96.9455

96.9460 (b)

High salinity, $1 \mathrm{~h}$ Up-regulated

High salinity, $1 \mathrm{~h}$ Down-regulated

Low salinity, $1 \mathrm{~h}$ Up-regulated

Low salinity, $1 \mathrm{~h}$ Down-regulated

High salinity, $5 \mathrm{~h}$ Up-regulated

Low salinity, $5 \mathrm{~h}$ Up-regulated

Low salinity, $5 \mathrm{~h}$ Down-regulated

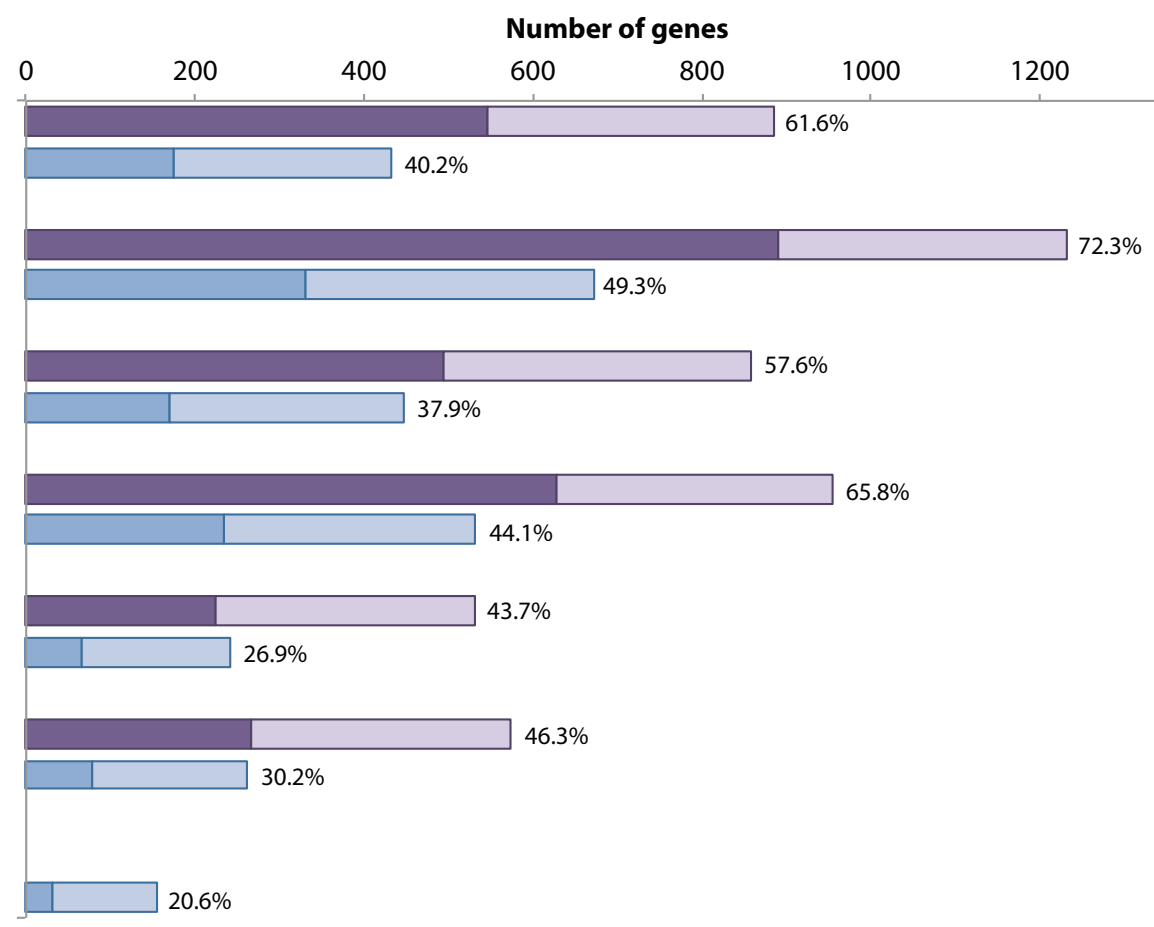

1400

genes in clusters; cutoff: $1.0 \mathrm{~L}_{2} \mathrm{fc}$

$\square$ genes not in clusters; cutoff: $1.0 \mathrm{~L}_{2} \mathrm{fc}$

$\square$ genes in clusters; cutoff: $1.5 \mathrm{~L}_{2} \mathrm{fc}$

$\square$ genes not in clusters; cutoff: $1.5 \mathrm{~L}_{2} \mathrm{fc}$ 
Figure 3
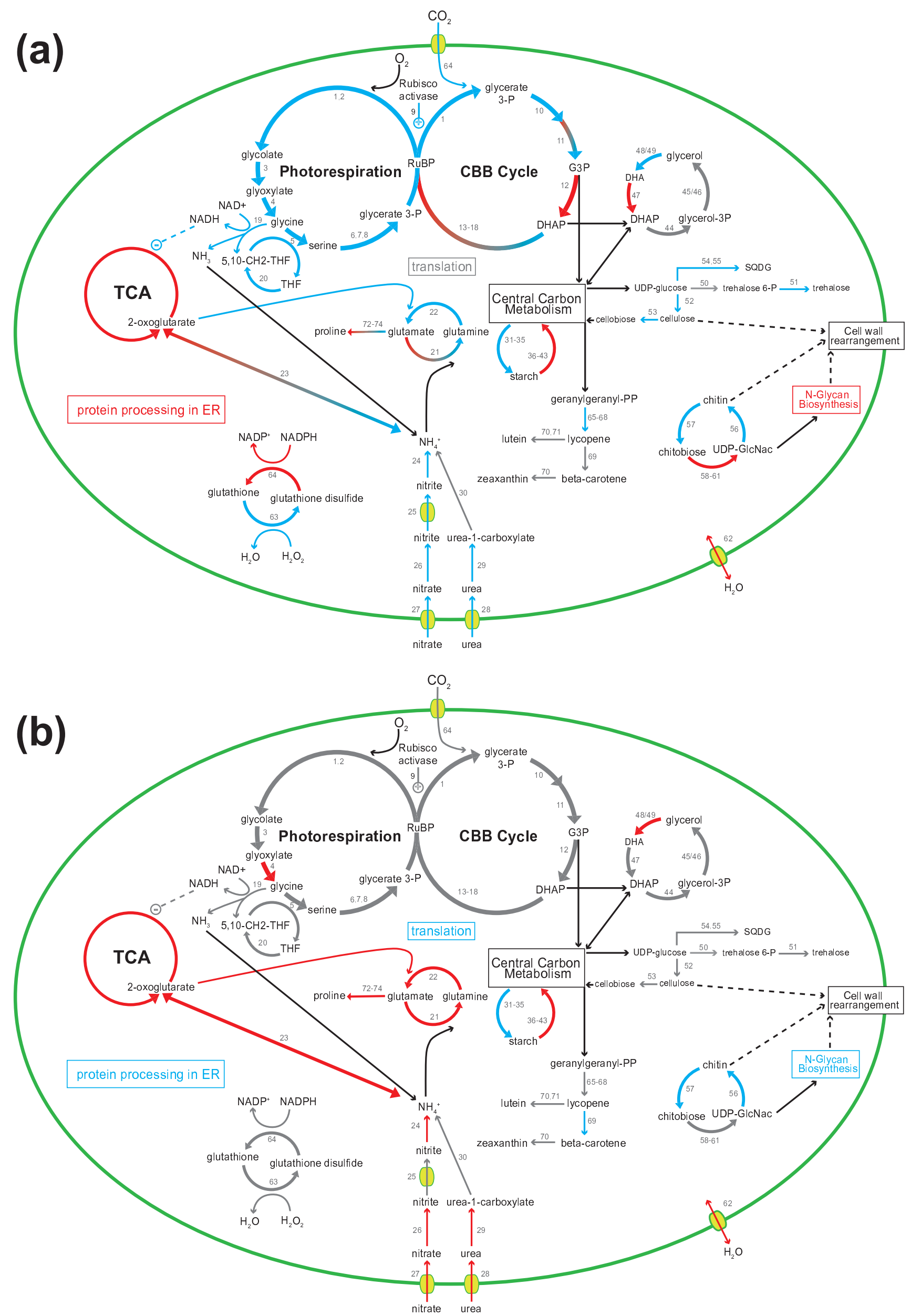

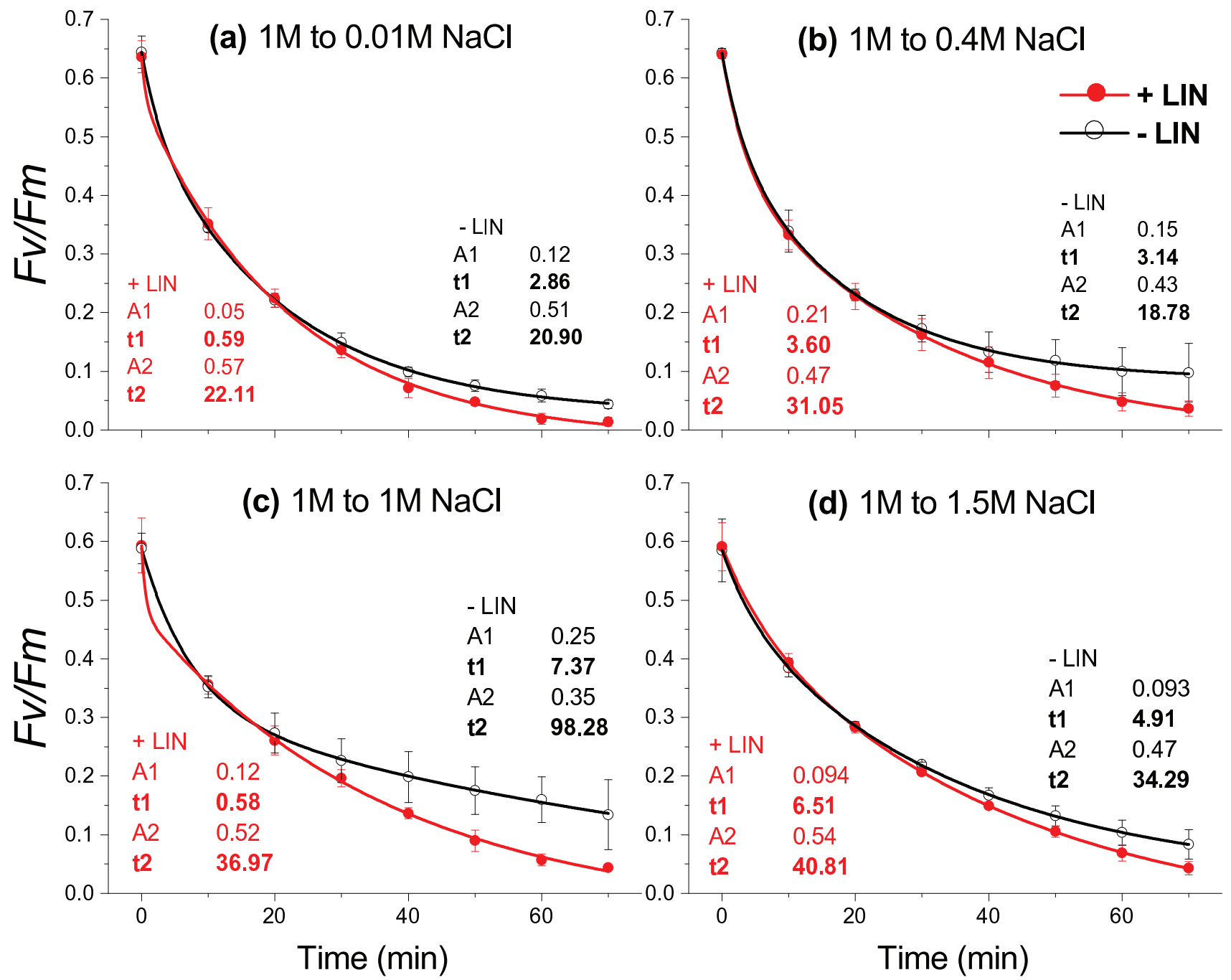